\title{
Ultrapiano: A Novel Human-Machine Interface Applied to Virtual Reality
}

\author{
Mikel Sagardia, Katharina Hertkorn, David Sierra González, and Claudio Castellini
}

In the quest for better human-machine interfaces (HMIs) for teleoperation and virtual reality, we hereby present the first integrated application of medical ultrasound imaging to remotely control a virtual piano playing environment in realtime.

A successful interaction with the remote environment is crucial for teleoperation systems. Thus, detecting human finger motions and forces plays an important role in preserving fine manipulation capabilities and hand dexterity. Standard kinematic devices, such as the CyberGlove, provide an accurate measurement assuming the glove being perfectly adherent to the subject's skin. Problems like intrinsic elasticity of the glove and different shapes of human hands limit their capabilities. Optical motion tracking systems, such as the Vicon system, allow for maximum freedom of motion (provided that markers are correctly set on the hand) but are prone to occlusion. Both kinds of systems need a long calibration procedure until the setup is ready to use.

We present medical ultrasound imaging as an accurate way of detecting human finger motions [1]. Recently, it has been shown that this technology can work in real-time and necessitates a short and easy calibration procedure [2]. In particular, finger forces can be individually predicted using forearm cross section ultrasound images. These forces are obtained in reference to the maximum stress applied during the calibration. The only sensor needed during the process is the ultrasound probe, which can be fixed on the ventral side of the forearm. This leaves the subject's hand completely free to operate.

We use this novel HMI to control piano playing in a virtual reality environment. Our virtual reality framework consists of a fast collision detection combined with a physics engine, the visualization tool InstantPlayer [3], and a sound player controlled by the collision forces. The collision detection is based on the Voxmap-Pointshell Algorithm [4], [5], which is suitable for haptic rendering since it computes collision forces within $1 \mathrm{~ms}$. The physics engine Bullet [6] is used to solve the motion equations under constraints. The combination of this physics engine with our collision detection framework enables realistic interaction with several complex virtual objects at haptic update rates.

Our integrated system can be used as an entertainment device, for rehabilitation of an impaired hand (e.g., in case of a stroke or of neuromuscular condition) and to aid the recovery from phantom-limb pain for amputees, along the

\footnotetext{
All authors are with the Robotics and Mechatronics Center, German Aerospace Center (DLR), Wessling, Germany. \{firstname.lastname\}edlr.de
}

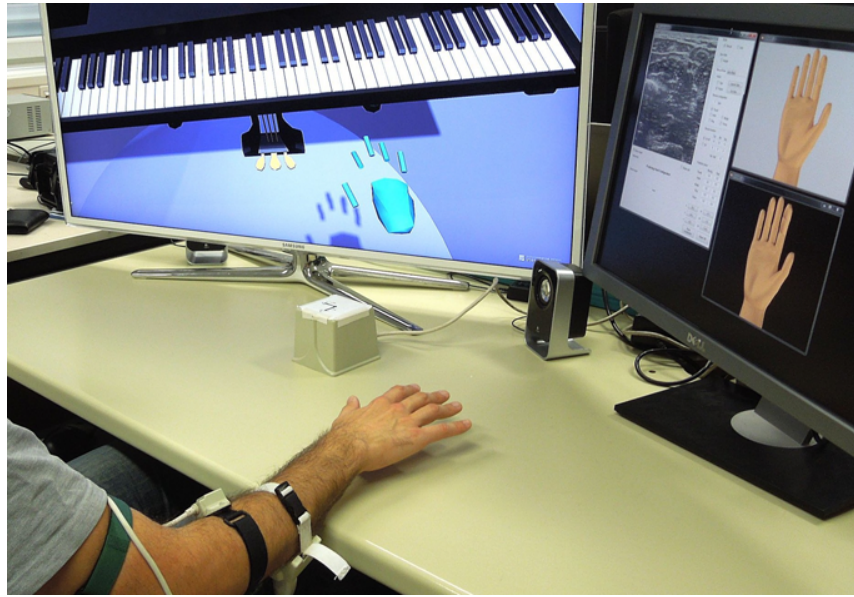

Fig. 1. The ultrapiano setup has four main components: an ultrasound machine and probe capturing images of the forearm, a finger force prediction system, a magnetic tracker to detect the hand movement and a realistic virtual environment.

lines of mirror therapy [7], [8].

Future work includes studying the interaction of the different fingers in order to be able to detect multi-fingered forces. The system is as well being optimized to reduce the total delay between the action of the subject and the response of the VR environment (currently around $300 \mathrm{~ms}$ ).

\section{REFERENCES}

[1] C. Castellini, G. Passig, and E. Zarka, "Using ultrasound images of the forearm to predict finger positions," IEEE Transactions on Neural Systems and Rehabilitation Engineering, vol. 20, no. 6, pp. 788-797, 2012.

[2] D. Sierra González and C. Castellini, "A realistic implementation of ultrasound imaging as a human-machine interface for upper-limb amputees," Frontiers in Neurorobotics, 2013, to appear.

[3] J. Behr, P. Dähne, Y. Jung, and S. Webel, "Beyond the web browser $\mathrm{x} 3 \mathrm{~d}$ and immersive vr," in Proc. of IEEE Symp. on 3D User Interfaces, 2007.

[4] W. A. McNeely, K. D. Puterbaugh, and J. J. Troy, "Voxel-based 6-dof haptic rendering improvements," Haptics-e: The Electronic Journal of Haptics Research, vol. 3, 2006.

[5] M. Sagardia and T. Hulin, "Fast and accurate distance, penetration, and collision queries using point-sphere trees and distance fields," in SIGGRAPH Posters, 2013, p. 83.

[6] "Bullet," 2013, accessed September 11th, 2013. [Online]. Available: http://bulletphysics.org/

[7] V. S. Ramachandran, D. Rogers-Ramachandran, and S. Cobb, "Touching the phantom limb," Nature, vol. 377, no. 6549, pp. 489-490, 1995

[8] B. L. Chan, R. Witt, A. P. Charrow, A. Magee, R. Howard, P. F. Pasquina, K. M. Heilman, and J. W. Tsao, "Mirror therapy for phantom limb pain," New England Journal of Medicine, vol. 357, no. 21, pp. 2206-2207, 2007. [Online]. Available: http://www.nejm.org/doi/full/10.1056/NEJMc071927 